\title{
3D RECONSTRUCTION OF A MULTISCALE MICROSTRUCTURE BY ANISOTROPIC TESSELLATION MODELS
}

\author{
Hellen Altendorf ${ }^{凶}, 1$, Felix Latourte ${ }^{2}$, DominiQue Jeulin ${ }^{1}$, Matthieu \\ FAESSEL $^{1}$ AND LUCIE SAINTOYANT ${ }^{2}$ \\ ${ }^{1}$ Centre of Mathematical Morphology, Mines Paris Tech, 35 rue Saint Honoré, 77305 Fontainebleau cedex, \\ France; ${ }^{2}$ EDF R\&D, Matériaux et Mécanique des Composants, Renardières, Moret-sur-Loing, France \\ e-mail: hellen.altendorf@mines-paristech.fr, felix.latourte@edf.fr, dominique.jeulin@mines-paristech.fr, \\ matthieu.faessel@mines-paristech.fr, lucie.saintoyant@edf.fr \\ (Received November 15, 2013; revised March 13, 2014; accepted April 17, 2014)
}

\begin{abstract}
In the area of tessellation models, there is an intense activity to fully understand the classical models of Voronoi, Laguerre and Johnson-Mehl. Still, these models are all simulations of isotropic growth and are therefore limited to very simple and partly convex cell shapes. The here considered microstructure of martensitic steel has a much more complex and highly non convex cell shape, requiring new tessellation models. This paper presents a new approach for anisotropic tessellation models that resolve to the well-studied cases of Laguerre and Johnson-Mehl for spherical germs. Much better reconstructions can be achieved with these models and thus more realistic microstructure simulations can be produced for materials widely used in industry like martensitic and bainitic steels.
\end{abstract}

Keywords: anisotropic tessellation, martensitic steel, multiscale 3D microstructure, stochastic modeling.

\section{INTRODUCTION}

This work has been motivated by the need of better understanding the fracturation of welding joints that is a general industrial issue faced in many applications. This phenomenon is known to be caused by a change of microstructure due to thermal differences arising during the welding process. To virtually simulate the influence of the microstructure changes to the mechanical response of a polycrystalline aggregate, we first need to achieve a reasonable stochastic model of its microstructure. The studies presented in this paper have mostly been validated on a grade 91 tempered martensitic steel, that is a $9 \%$ chromium steel yielding a multiscale microstructure. In this paper, the two largest scales of the microstructure have been considered: the austenitic grains and the packets inside each grain, which are modeled by a two-step random tessellation model.

The most important and best known tessellation models are Voronoi (Descartes, 1644; Dirichlet, 1850; Voronoi, 1908), Laguerre also called power diagrams (Blaschke, 1921; Lautensack, 2007) and JohnsonMehl (Johnson and Mehl, 1939). All three models make use of an isotropic growth model. The Voronoi tessellation builds on a random point process and creates the region by an isotropic growth starting from the point germs. The Laguerre and JohnsonMehl tessellations assign a random radius to each point of the random point process and thus are built on spherical germs. The Laguerre mosaic starts the growth from the sphere boundary and the JohnsonMehl mosaic proceeds a time delayed growth, due to a continuous germination process.

The originality of this work is to introduce new anisotropic growth models and to combine them to a two-step tessellation model . Furthermore, we show that the new approaches outperform the aforementioned models for the considered microstructures. In a first step, the austenitic grains are modeled by a Laguerre tessellation and in a second step the regions of the austenitic grains are tessellated by an anisotropic growth model from ellipsoidal shaped implanted germs. Compared to the quite complex shapes of the packets this model is relatively simple and allows to reach a certain level of abstraction that is desirable when optimal trade-offs between realism and complexity are needed. Therefore, we also introduce a model to reconstruct the packet in any required precision and compare the quality and use-cases of both models. The second model is a reunion of Laguerre regions by successively implanted maximal spheres. This model is especially useful for abstraction of a real mosaic structure, allowing a relatively simple surface or volume meshing to be created with a required precision or abstraction level.

The following of the paper is structured in three sections: Materials and methods, Results, and Discussion. In Materials and methods, we explain in details the material of interest and the available images 
as well as the developed tessellation models. The following section shows the results on the applied data sets and the last section discusses the advantages and limitations of the method.

\section{MATERIALS AND METHODS}

In this section, we introduce the material of interest and the imaging technique. We explain in details the multiscale microstructure at the level of grains and packets that is analyzed and mention the information that is available due to the imaging technique. In the third subsection, we describe in details the stochastic model to reconstruct the microstructure decomposed into grains and packets.

\section{MATERIAL}

The materials of interest for our studies are grade P91 and P92 steels used in components of nuclear power stations of generation IV and thermal power stations, and also in steam circulations (operated under conditions with high pressure and temperature). This steel yields a martensite microstructure and is used due to its good creep resistance, its low thermal expansion compared to austenitic steels and its weldability. The welded joints obtained are susceptible to type IV cracking, that mainly occurs in the fine grained heat affected zone (FGHAZ) or inter-critical heat affected zone (ICHAZ) also having fine grains (Albert et al., 2003). One of the main reasons for this susceptibility is the heterogeneity of the microstructure near the FGHAZ / ICHAZ which results in a contrast of mechanical properties and causes a localization of damage and deformation in the area FGHAZ / ICHAZ.

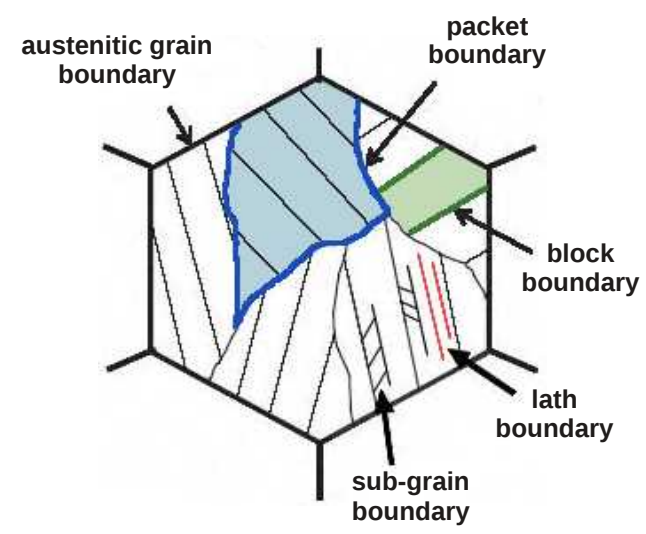

Fig. 1. Microstructure of steel P91 according to Vivier (2009).
Fig. 1 illustrates the multiscale microstructure of steel P91. Notice that the austenitic grains are schematically idealized as a hexagon, while in the real material the structure is more complex but can still keep a relatively regular and nearly convex shape. We concentrate in this work on the grain and packet scale, which are the easiest shapes to extract from experimental crystallographic orientation images. Notice that the segmentation of the austenitic grains is a rather difficult quest and cannot be easily undertaken using an automated algorithm.

\section{IMAGES}

This study is based on image data required by "electron backscatter diffraction" (EBSD). EBSD also known as orientation imaging microscopy, makes use of the backscattered diffraction pattern from the beam of a scanning electron microscope. This pattern yields information on the rotation of the crystal lattice, a quality factor (IQ) defining the sharpness of the diffraction pattern, the "confidence index" indicating the degree of confidence that the orientation calculation is correct, Hough data, the phase of the material, and the location where the data was obtained on the specimen.

The angular and spatial resolution of EBSD data easily allows to segment and label packets, while laths having boundaries of low misorientation (typically less than two or three degrees) and having much smaller dimensions are more difficult to extract. In the IQ image, we observe that there is a block structure visible (Fig. 2b), that is not considered in this work. We focus here on two crystalline scales: the former austenitic grains whose average size is about one hundred microns, and the packets, a few microns thick and directly visible in inverse pole figures (Fig. 2a). These two scales allow to understand and model significant microstructure variations between different heat affected zones of the weld, and therefore will be associated with different stress localization trends important to later understand while the crack initiates between FCHAZ and ICHAZ.

\section{METHOD}

In this section, we present three types of tessellation models: the classical isotropic growth models (Voronoi, Laguerre and Johnson-Mehl), the reunion of classical isotropic cells and the more abstract anisotropic growth model. The final part describes how these models are put together to multiscale tessellations. Troughout this section, we present some reconstructions of microstructures of martensitic and bainitic steel. These reconstructions 
show how good an existing microstructure can be presented with a stochastic model. This part does not correspond to the stochastic modeling process, but helps to choose the most realistic stochastic model for a given microstructure type. The fitting of the stochastic model includes the parameter estimation of the underlying point process and orientation and size distribution of the generating germs, which is not in the scope of this paper.

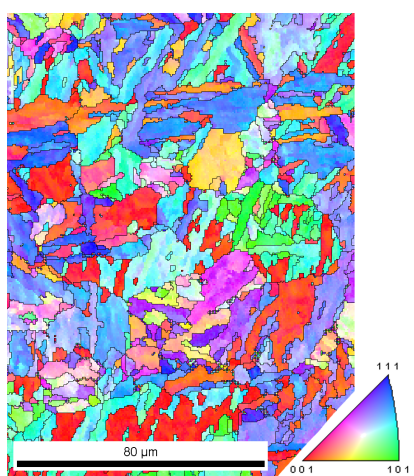

(a) Inverse Pole Figure

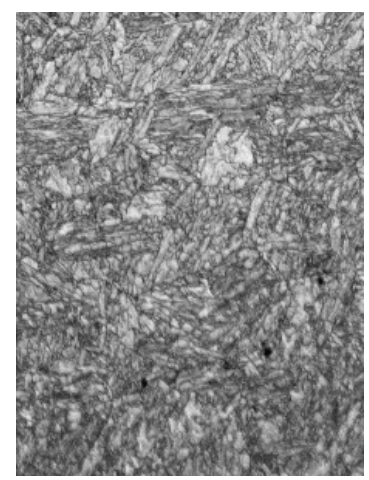

(b) Image Quality
Fig. 2. EBSD Imaging: Examples on a sample of base metal for (a) the inverse pole figure, representing the orientation of the normal direction in color, (b) the image quality (IQ) reveals lath and subgrain structures, that are not accounted for in our microstructure description. The size of the image is $225 \times 300$ pixels $[\equiv 112.5 \times 150 \mu \mathrm{m}]$ with a resolution of $0.5 \mu \mathrm{m} /$ pixel.

\section{Isotropic Growth Models}

The most common tessellation models are Voronoi, Laguerre and Johnson-Mehl. These three types of models are built on an isotropic growth model from a set of point germs $P=\left[p_{1}, \ldots, p_{n}\right]$, created by a point process in a domain $D \subset \mathbb{R}^{d}$. The cells are then defined by all points closest to a creating germ $p_{i}$ under a specific distance measure. Those cells are mathematically defined by

$$
C(i \mid P, D)=\left\{x \in D \mid d\left(x, p_{i}\right)<d\left(x, p_{j}\right), \forall j \neq i\right\} .
$$

The definition of the distance measure makes the difference of the three models. For the Voronoi tessellation the distance measure is the Euclidean distance:

$$
d_{\mathrm{V}}\left(x, p_{i}\right)=\left\|x-p_{i}\right\| .
$$

For the Laguerre and Johnson-Mehl tessellation, we furthermore assign a radius $r_{i}$ to each point $p_{i}$. The radius will be taken into account and the distance measure is replaced by a power.
For Laguerre:

$$
d_{\mathrm{L}}\left(x, p_{i}, r_{i}\right)=\left\|x-p_{i}\right\|^{2}-r_{i}^{2}
$$

and for Johnson-Mehl:

$$
d_{\mathrm{JM}}\left(x, p_{i}, r_{i}\right)=\left\|x-p_{i}\right\|-r_{i} .
$$

The differences in the distance measures give the mosaic different cell shapes. According to Lautensack (2007), these tessellation can be interpreted as follows. In the case of the Laguerre tessellation, the power distance has the following geometric interpretation: For each point $x \in \mathbb{R}^{d}$ outside the sphere $s\left(p_{i}, r_{i}\right)$ the value $d_{L}\left(x, p_{i}, r_{i}\right)$ equals the squared length of the tangent line from $x$ to the sphere. The JohnsonMehl tessellation can be interpreted as the result of a growth process of seeds being generated according to a random process in time.

The Johnson-Mehl tessellation creates curved cell boundaries, while the Voronoi and Laguerre models built on an isotropic growth and always create convex cells with straight boundaries.

In the real microstructure, we observe that the regions of the austenitic grains are relatively homogeneous and nearly convex. This indicates that it should be possible to represent them using an isotropic mosaic model. A first way to evaluate a model for a given microstructure is to assess how well the microstructure can be represented using this model description. For 2D mosaics a list of germs can be defined by the barycenters of each region and the radius $r_{i}$ computed from its area $S_{i}$ as follows: $r_{i}=\sqrt{S_{i} / \pi}$. The application of this approach to the labeling of the austenitic grains is shown in Fig. 3.

We observe that the sizes of the Voronoi cells are too homogeneous in comparison to the real grains, and the grain size distribution in the microstructure is poorly reproduced. An improvement to represent the original cells is noticed with the Laguerre and Johnson-Mehl cells, as these two models take into account the size of the regions. The difference between the last two mosaics is the shape of the boundaries. Johnson-Mehl introduces a curvature that seems favorable to respect the dimension of the smaller regions. Another consequence is that the curvature between two cells of different sizes is convex in the smaller cell and concave in the larger cell. This effect is not completely supported by the shapes of the real grains. 


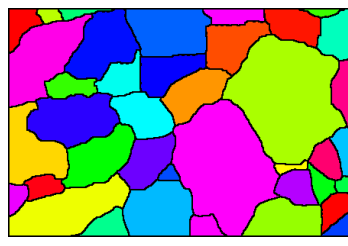

(a) Grain Labeling

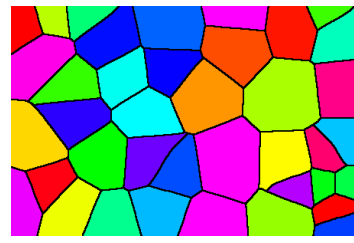

(c) Voronoi Mosaic

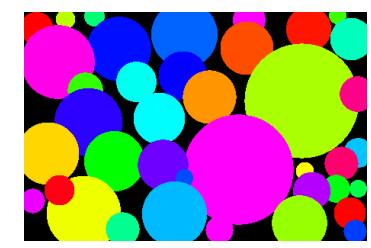

(b) Creating Disc-Germs

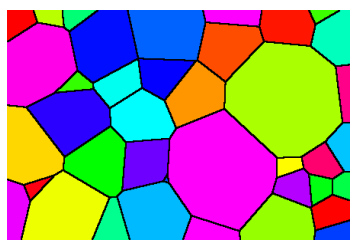

(d) Laguerre Mosaic

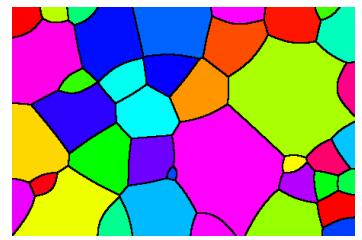

(e) Johnson-Mehl Mosaic

Fig. 3. Model reconstruction of the manual grain segmentation as Voronoi, Laguerre and Johnson-Mehl mosaic.

Concerning the modeling of the packets, the first guess is of course to implant similar mosaics in the grain cells. Fig. 4 shows a reconstruction of the packet regions as a Laguerre mosaic. It is quite obvious that the structure of a Laguerre Mosaic is much too regular to represent the packet regions. In particular, a Laguerre mosaic is only composed of convex cells, where the regions of the packets are highly nonconvex.

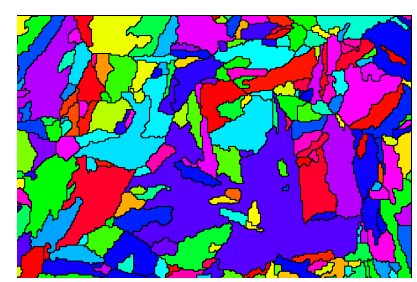

(a) Packet Labeling

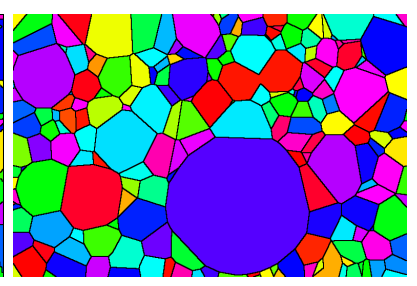

(b) Laguerre Mosaic
Fig. 4. Model reconstruction of the packet segmentation as Laguerre mosaic.

\section{Reconstruction as Reunion of Cells}

As simple isotropic mosaics are too regularly shaped, it becomes natural to test a model of increased complexity. One option is to create several smaller Laguerre cells and merges them to larger regions with more complex shapes to represent one packet.
The existing segmentation can be reconstructed by implanting iteratively maximal spheres/discs down to a certain size limit and build the reunion of cells with the same label. Fig. 5 shows the reconstruction of the packet segmentation as such a reunion of Laguerre cells.

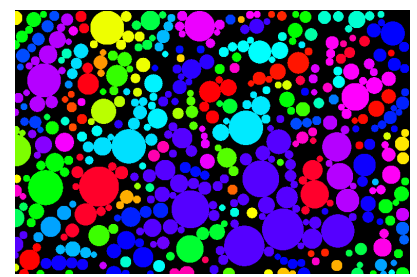

(a) Maximal Discs $R_{\min }=6$

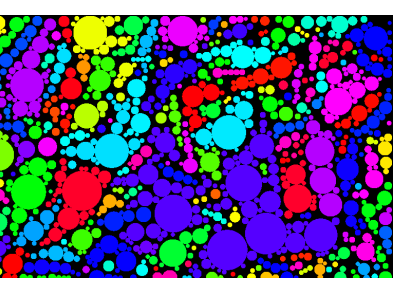

(c) Maximal Discs $R_{\min }=4$

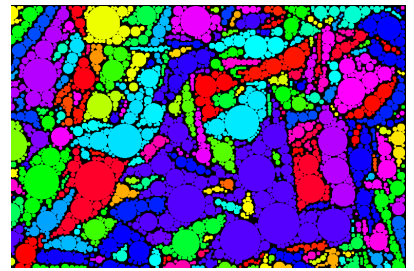

(e) Maximal Discs $R_{\min }=2$

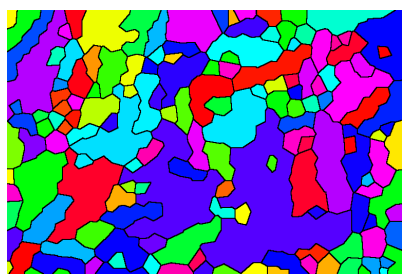

(b) Merged Laguerre $R_{\min }=6$

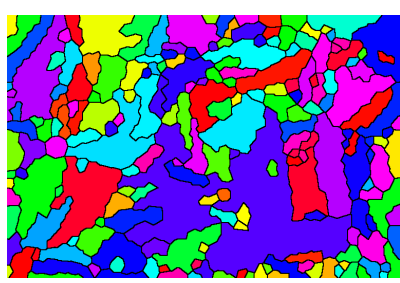

(d) Merged Laguerre $R_{\min }=4$

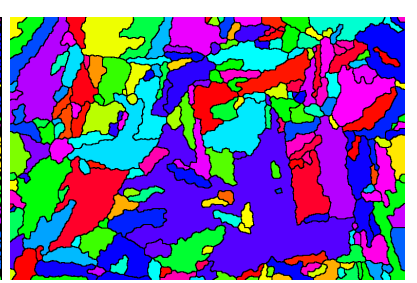

(f) Merged Laguerre $R_{\min }=2$
Fig. 5. Model reconstruction of the packet segmentation (shown in Fig. 4) as merged Laguerre mosaic from multiple maximal discs with different thresholds for the minimal disc radius $R_{\min } \in\{2,4,6\}$.

The precision of this reconstruction can be chosen with the use of a threshold for the radius of the spheres/discs. In this example, we show different choices of the minimal sphere/disc radius $R_{\min } \in$ $\{2,4,6\}$. We observe that for $R_{\min } \in\{4,6\}$ there are some missing regions in the reconstruction as the maximal sphere/disc that can be placed in small regions has a smaller radius than the $R_{\min }$ threshold. Therefore, these small regions are interpreted as noise and not reconstructed for the required level of abstraction. For $R_{\min }=2$ the regions seems perfectly reconstructed. At first this might seem relevant, however this strategy suffers from a lack of abstraction. The regions are reconstructed with every little details, and an artificial roughness of the boundaries is remodeled even if it is caused by discretization artifacts. Therefore, the threshold $R_{\min }=4$ gives the best trade-off between precision and abstraction. 
This model seems to be very promising in the sense that a given microstructure can be reconstructed as a model with any given precision, adjustable with the minimal radius parameter. Still, the main goal to generate new random structures with similar characteristics demands for rules to create a stochastic model with assessable parameters. For this model, the rules need to describe how to merge mosaic cells to different packet regions. This step is highly non trivial and not very promising for realistic descriptors and reconstructions. Therefore, we propose in the following anisotropic tessellation models with a higher level of abstraction.

\section{Anisotropic Tessellations}

In the case of more complex cell shape, we need to create a tessellation model that generates non convex and anisotropic cell shapes. Jeulin (2013) proposes local metrics for mosaic models in the form of symmetric positive definite matrices $M_{i}$ and centers $c_{i}$. The local metric of cell $i$ is then defined by:

$$
d\left(x, c_{i}\right)=\left(x-c_{i}\right)^{T} M_{i}\left(x-c_{i}\right) .
$$

The anisotropic growth is defined by the eigenvectors and eigenvalues of the matrix $M_{i}$ and the tessellation can be interpreted as an ellipsoidal germ process, where the eigenvectors describe the ellipsoidal axes and the eigenvalues define their lengths. Based on those ellipsoidal germs, we can define different metrics that affect the tessellation boundaries of the cells, assuming a cell is built up by all points having the closest distance to a specific germ. Thus the cell $C_{i}$ around an ellipsoidal germ $e_{i}$ is defined as:

$$
C_{i}=\left\{x \in \mathbb{R} \mid d\left(x, e_{i}\right) \leq d\left(x, e_{j}\right) \forall j \in I\right\},
$$

where $d$ is an arbitrary metric and $I$ the index space of the germs. A list of possibilities is shown in Table 1 and the corresponding boundaries for two germs are visualized in Fig. 6. The definition of $d_{e}$ corresponds to the matrix definition given in Eq. 5 .

In Table 1, the corresponding germ is an ellipsoid with axes $v_{x}, v_{y}, v_{z}$ and according radii $r_{x} \geq r_{y} \geq r_{z}$. The coordinates $\left(x^{\prime}, y^{\prime}, z^{\prime}\right)$ are the rotated coordinates of the point $p$, defined by the ellipsoid rotation such that $v_{x}$ is transformed to $(1,0,0)^{T}$ etc. Further definitions are used: the volumetric radius $r_{v}$ is the radius of a sphere/disc with equal volume than the ellipsoid: $V_{e}=$ $V_{s}\left(r_{v}\right) \Rightarrow r_{v}=\sqrt[3]{r_{x} r_{y} r_{z}}$ and the specific radius $r_{s}(p, e)$ is the width of the ellipsoid in direction $p-c$ between the point of interest $p$ and the ellipsoidal center $c$. (s) and (ns) defines the squared and non-squared versions.
Table 1. Definition of local metrics for an anisotropic ellipsoidal germ process.

\begin{tabular}{|ll|}
\hline Model & Distance Function \\
\hline ellipsoid equation (s) & $d_{e}^{2}(p, e)=\frac{x^{2}}{r_{x}^{2}}+\frac{y^{\prime 2}}{r_{y}^{2}}+\frac{z^{2}}{r_{z}^{2}}$ \\
normalized (s) & $d_{1}(p, e)=d_{e}^{2}(p, e)-1$ \\
normalized (ns) & $d_{2}(p, e)=d_{e}(p, e)-1$ \\
max. radius (s) & $d_{3}(p, e)=r_{x}^{2}\left(d_{e}^{2}(p, e)-1\right)$ \\
max. radius (ns) & $d_{4}(p, e)=r_{x}\left(d_{e}(p, e)-1\right)$ \\
min. radius (s) & $d_{5}(p, e)=r_{z}^{2}\left(d_{e}^{2}(p, e)-1\right)$ \\
min. radius (ns) & $d_{6}(p, e)=r_{z}\left(d_{e}(p, e)-1\right)$ \\
vol. radius (s) & $d_{7}(p, e)=r_{v}^{2}\left(d_{e}^{2}(p, e)-1\right)$ \\
vol. radius (ns) & $d_{8}(p, e)=r_{v}\left(d_{e}(p, e)-1\right)$ \\
isotr. growth (s) & $d_{9}(p, e)=\|p-c\|^{2}-r_{s}(p, e)^{2}$ \\
isotr. growth (ns) & $d_{10}(p, e)=\|p-c\|-r_{s}(p, e)$ \\
specific rad. (s) & $d_{11}(p, e)=r_{s}^{2}\left(d_{e}^{2}(p, e)-1\right)$ \\
specific rad. (ns) & $d_{12}(p, e)=r_{s}\left(d_{e}(p, e)-1\right)$ \\
\hline
\end{tabular}

The difference between these new definitions and the matrix approach is that the metrics are designed to correspond to the classical Laguerre and JohnsonMehl tessellation for the spherical case $r_{x}=r_{y}=$ $r_{z}$. The metrics $d_{3}, d_{5}, d_{7}, d_{9}, d_{11}$ degenerate to the Laguerre tessellation and $d_{4}, d_{6}, d_{8}, d_{10}, d_{12}$ to the Johnson-Mehl tessellation. As shown in Fig. 6. The metrics $d_{e}, d_{1}, d_{2}$ correspond to the same boundaries, $d_{1}$ and $d_{2}$ were just taken into account as they build the basis for the following metrics. Proof of the equivalence between these metrics can be found in the appendix.

Furthermore, we observe that the squared (s) and non-squared (ns) versions do not differ much from each other. And even if most of the squared version correspond to the Laguerre tessellation (with straight/planar boundaries) in the spherical case, they do not have straight boundaries in the non-spherical case.

The anisotropic tessellation can be fitted to an existing mosaic by evaluating the inertia moments and axes of each region. The eigenvectors of the covariance matrix of a region are linearly related to the radii of the corresponding ellipsoid (McCartin, 2007). Figs. 7 and 8 show the comparison of different tessellation models to the grain and packet mosaic. The fit of a model is evaluated by the ratio of false labeling, which is defined as the amount of false labeled pixels divided by the amount of all pixels. This evaluation is shown in Table 2. This evaluation shows the best results in the present case for the ellipsoidal germs with anisotropic local metric $d_{5}$, directly followed by the results obtained for metric $d_{e}$. 

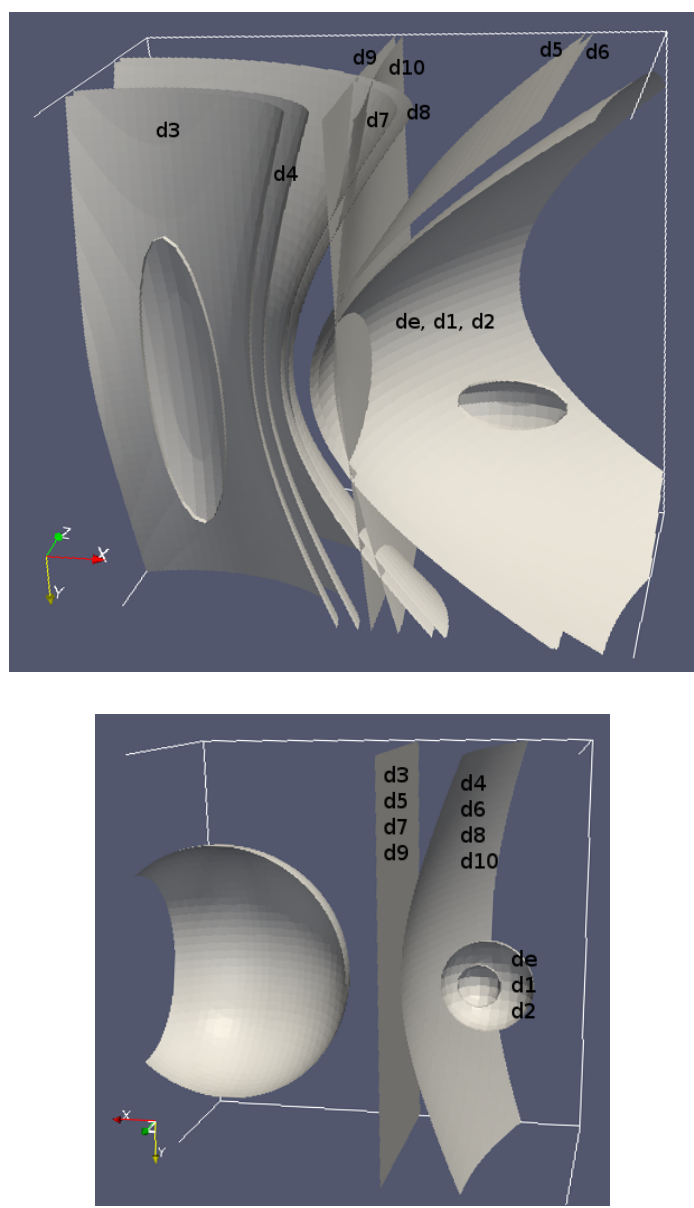

Fig. 6. The boundaries of an anisotropic tessellation with various metric definitions (defined in Table 1). Top: with two orthogonal ellipsoids as germs, bottom: with two spherical germs. Notice that in the spherical case the boundaries $d 3, d 5, d 7$, and $d 9$ coincide with the Laguerre tessellation and the boundaries $d 4, d 6$, $d 8$, and d10 with the Johnson-Mehl tessellation.

Table 2. False labeling of model fits: ratio of false labeled pixels divided by the amount of all pixels.

\begin{tabular}{lrr}
\hline Model & Grain Seg. & Paket Seg. \\
\hline Voronoi & $30.56 \%$ & $51.18 \%$ \\
Laguerre & $15.44 \%$ & $39.96 \%$ \\
JohnsonMehl & $13.64 \%$ & $35.47 \%$ \\
\hline Ellipsoid $d_{e}$ & $7.01 \%$ & $13.86 \%$ \\
Ellipsoid $d_{3}$ & $10.58 \%$ & $23.35 \%$ \\
Ellipsoid $d_{4}$ & $8.39 \%$ & $18.84 \%$ \\
Ellipsoid $d_{5}$ & $6.90 \%$ & $13.17 \%$ \\
Ellipsoid $d_{6}$ & $6.94 \%$ & $13.45 \%$ \\
Ellipsoid $d_{7}$ & $8.86 \%$ & $19.55 \%$ \\
Ellipsoid $d_{8}$ & $7.66 \%$ & $16.48 \%$ \\
Ellipsoid $d_{9}$ & $10.93 \%$ & $24.68 \%$ \\
Ellipsoid $d_{10}$ & $8.47 \%$ & $19.52 \%$ \\
Ellipsoid $d_{11}$ & $10.92 \%$ & $24.68 \%$ \\
Ellipsoid $d_{12}$ & $8.47 \%$ & $19.52 \%$ \\
\hline
\end{tabular}

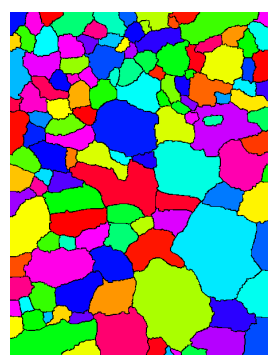

(a) Grain Segm

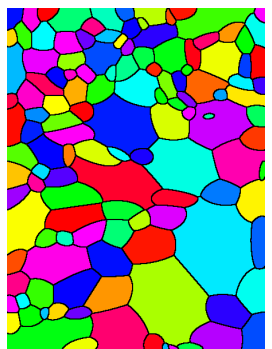

(d) Ell. Tess. d0

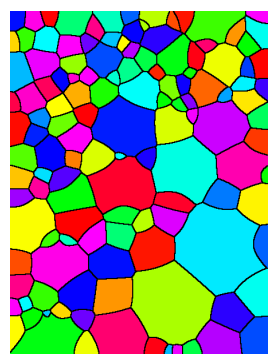

(b) JohnsonMehl

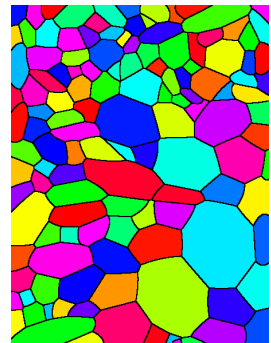

(e) Ell. Tess. d3

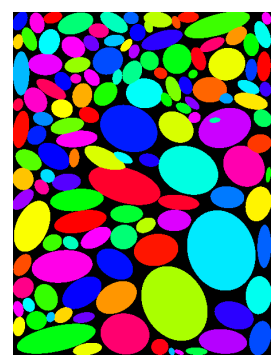

(c) Ellipses

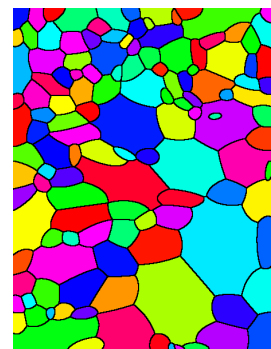

(f) Ell. Tess. d5
Fig. 7. Fitting of the tessellation models to the manual segmentation of the austenitic grains.

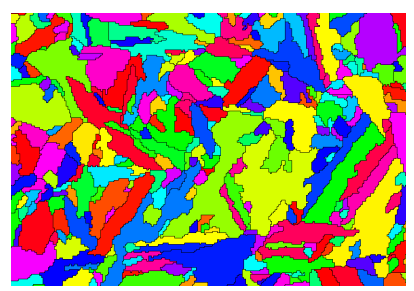

(a) Packet Segm.

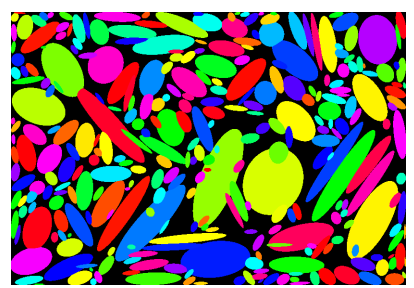

(c) Ellipses

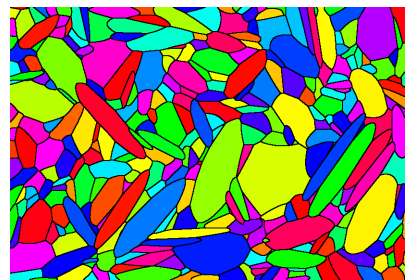

(e) Ell. Tess. d3

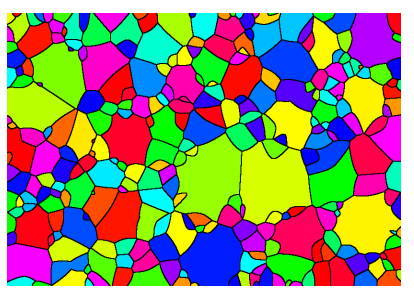

(b) JohnsonMehl

(d) Ell. Tess. d0

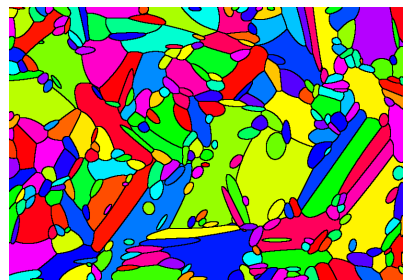

(f) Ell. Tess. d5

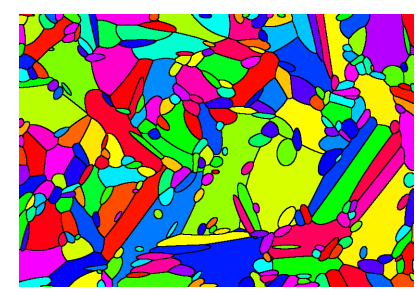

Fig. 8. Fitting of the tessellation models to the packet mosaic. 


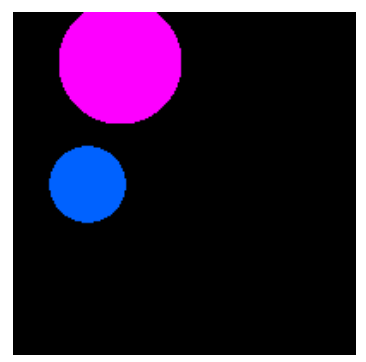

(a) Spherical Germs

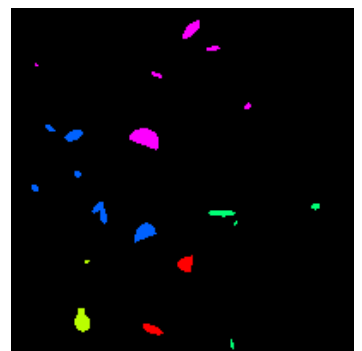

(c) Ellipsoidal Germs

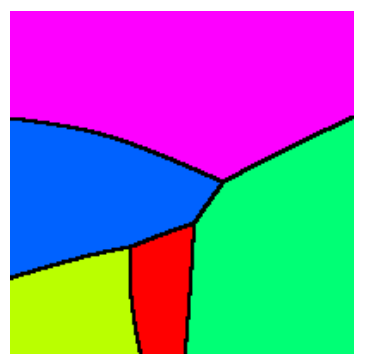

(b) Grains

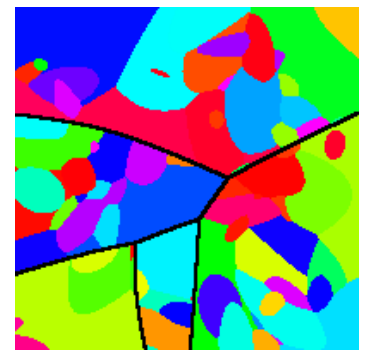

(d) Packets
Fig. 9. Examples of an two-scales tessellation tessellation model. We show here a slice of a 3D model.

\section{Multiscale Tessellation}

Finally, the tessellation models are integrated in a multiscale tessellation model by reducing the domain in each step to a cell of a higher tessellation order. The first order in this example are the austenitic grains $C\left(G_{i}\right) \subset W \subset \mathbb{R}^{3}$ (for $i \in I_{G}$ ) as a result of a JohnsonMehl tessellation in the window $W$ with $n$ spherical germs $G_{i}$ indexed with $I_{G}$. In the second step we create ellipsoidal germs $P_{j}$ in each cell $C\left(G_{i}\right)$ (where only the center, and not the complete ellipsoids need to be part of $C\left(G_{i}\right)$ ). Those ellipsoidal germs create the packets with $C\left(P_{j} \mid G_{i}\right)=\left\{x \in C\left(G_{i}\right) \mid d\left(x, P_{j}\right)<d\left(x, P_{k}\right) \forall k \in\right.$ $\left.I_{P, i}\right\}$. Thus we, build the tessellation in $\mathbb{R}^{3}$ and intersect it with the given domain $C\left(G_{i}\right)$. Fig. 9 shows an example of such a 3D multiscale tessellation. This describes only a two-scale tessellation, but this process can be iterated to any arbitrary scale multiplicity.

\section{RESULTS}

This section shows some three-dimensional examples for the anisotropic growth tessellations, that points out the characteristics of non-conncected cells. Furthermore, we evaluate the goodness of reconstruction for some samples of martensitic and bainitic steel.

Fig. 10 shows two examples of anisotropic tessellations with a boolean model of ellipsoidal germs and local metric $d_{e}$. This kind of tessellation produces non convex cells elongated in the main ellipsoidal direction $v_{x}$. Furthermore, it might happen that a cell is non connected. An example of this case is shown in Fig. 11, where the upper left cell belongs to the lower right ellipsoid and the upper right cell belongs to the lower left ellipsoid.
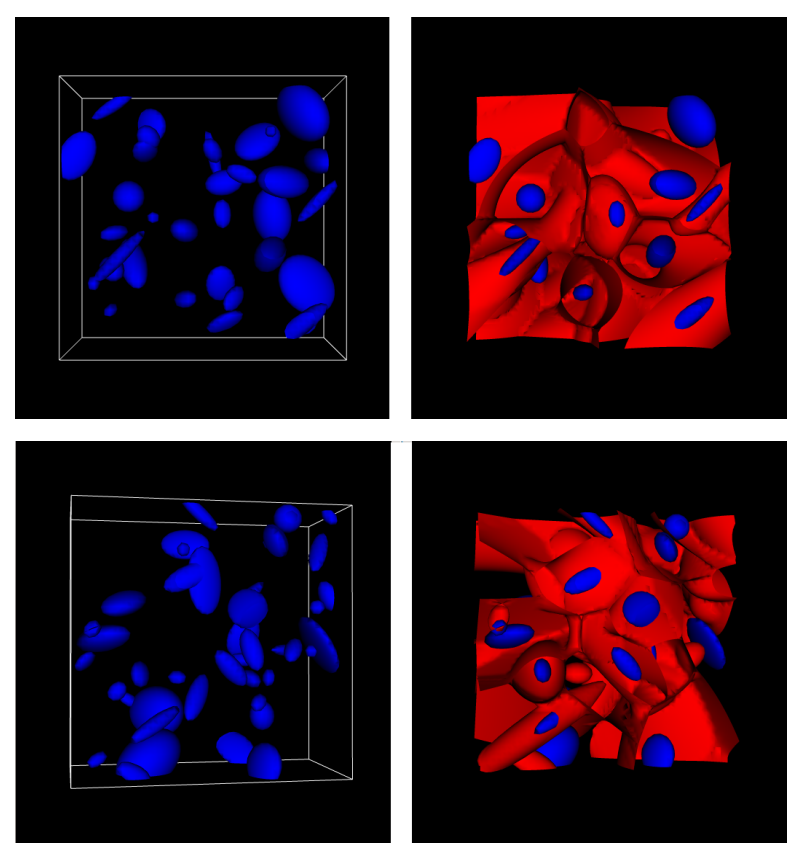

Fig. 10. Examples of an anisotropic tessellation with boolean ellipsoid germs and the local distance measure $d_{e}$

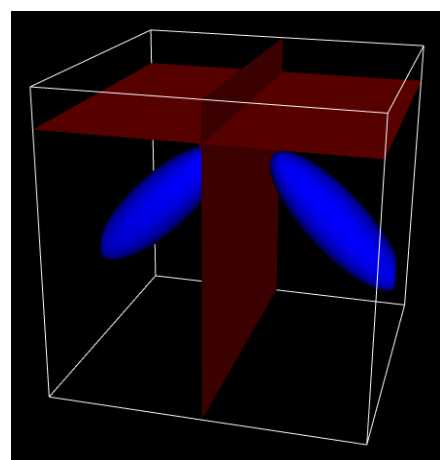

Fig. 11. Example of non connected cells, generated from the same germ.

The following results are reconstructions of several EBSD images from different heat affected zones of a martensitic steel. The evaluations are shown in the first part of Table 3. The same procedure is also applied on the 3D data sets of martensitic and bainitic steels as shown in Fig. 12. The example shows that the model initially developed for martensitic steel can also be applied to bainitic steel. 
Table 3. Statistics on reconstructed anisotropic tessellations. For each data set, the table shows the amount of germs and the false labeling for the $d_{e}$ function and the minimal false labeling of the remaining distance measures. Furthermore, we mention the distance measure with the lowest false labeling.

\begin{tabular}{lrrr}
\hline Zone & Germs & \multicolumn{2}{c}{ False Labeling } \\
& & $d_{e}$ & min. \\
\hline BM & 1904 & $13.55 \%$ & $12.95 \%\left(d_{5}\right)$ \\
ICHAZ & 1982 & $11.65 \%$ & $11.27 \%\left(d_{5}\right)$ \\
FGHAZ & 1600 & $12.15 \%$ & $11.92 \%\left(d_{5}\right)$ \\
CGHAZ & 1704 & $14.70 \%$ & $14.27 \%\left(d_{6}\right)$ \\
WM & 1612 & $15.69 \%$ & $15.19 \%\left(d_{6}\right)$ \\
\hline 3D martensitic s. & 878 & $24.74 \%$ & $28.67 \%\left(d_{4}\right)$ \\
3D bainitic steel & 216 & $24.75 \%$ & $32.29 \%\left(d_{6}\right)$ \\
\hline 3D simulation & 23 & $19.28 \%$ & $18.85 \%\left(d_{6}\right)$ \\
\hline
\end{tabular}
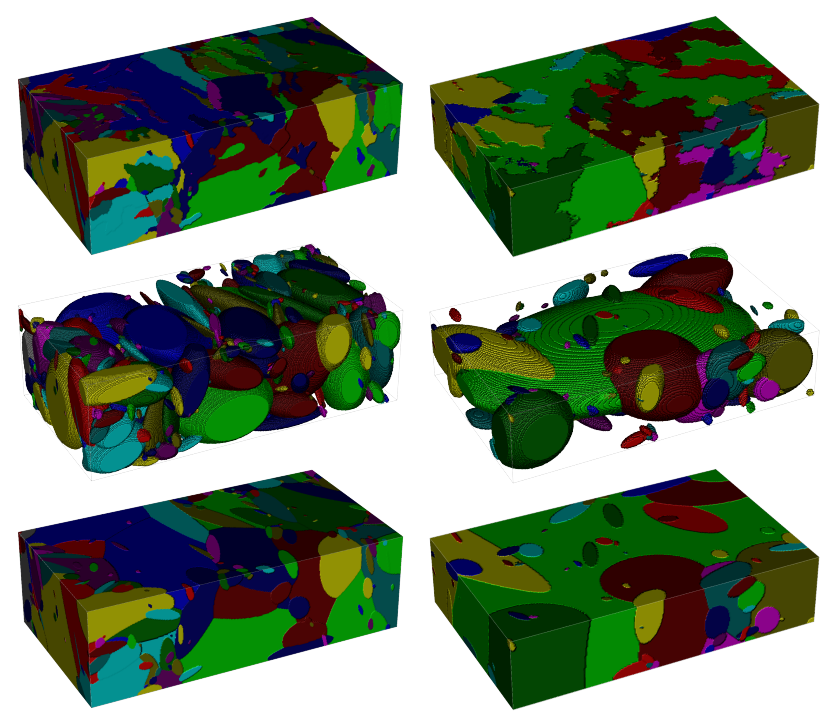

Fig. 12. Reconstruction of $3 D$ packet structure with the anisotropic tessellation model based on ellipsoidal germs. Left: $3 D$ Base metal of an martensitic steel P91; Right: tempered bainitic steel A508cl3 (16MND5 in French) used for the construction of the pressure vessel of French pressurized water reactors. Row 1: labeled packet tessellation, row 2: ellipsoidal germs, row 3: reconstruction tessellation by inertia moments.

Table 3 shows the amount of packets and the false labeling in percent, which is the relative amount of pixels receiving a different label in the reconstruction than in the original label mosaic. The 2D reconstruction shows false labeling reaching from $10 \%$ to $15 \%$, which is a very good result. Note that the false labeling should be optimally low, although $0 \%$ would mean a complete reconstruction of the region.
Such a complete reconstruction is achievable using for example the multiple sphere model with radii down to 1 pixel resolution. Such a perfect reconstruction is however not very interesting as it reconstructs all noisy deviations at the boundaries, some level of abstraction and of simplification being requested.

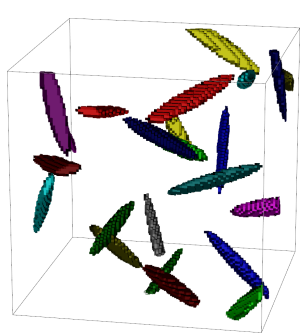

(a) Simulated ell. germs

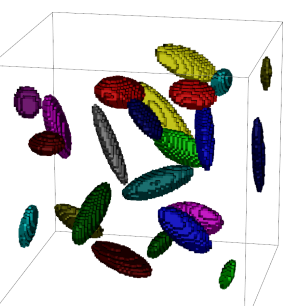

(c) Inertia ellipsoids downscaled

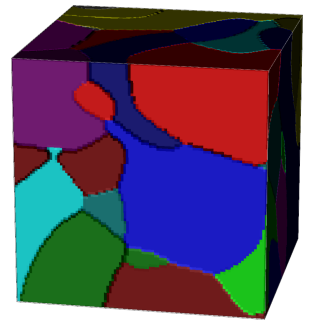

(b) Tessellation to (a)

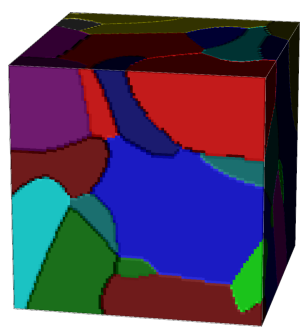

(d) Tessellation to (c)
Fig. 13. Reconstruction of a simulation of a $3 D$ anisotropic tessellation model based on random ellipsoid germs. Thus the ellipsoid germs of (c) are downscaled for a better visual comparison to (a). Note that a scaling of the ellipsoid radii by a constant factor does not influence the tessellation.

\section{DISCUSSION}

In Fig. 10, we observed that crossing cells can be generated, although is not very common. In general, the underlying creating process of the ellipsoidal germs follows some orientation distribution or a rather dense packing algorithm. Thus, most neighboring cells have a similar elongation and the crossing lies so far away, that another germ generates a grain before the crossing might occur.

In the 3D reconstruction examples, the false labeling rises to about $25 \%$. This decrease in the quality of reconstruction could be due to a more complex structure that cannot be reconstructed in a very precise matter by the chosen model. It can also result from edge effects: due to the relatively low size of the 3D images as compared to the size of packets, many of them being cut by the boundaries of the domain. Still, also the reconstruction of tessellations 
from an ellipsoidal germ model (Fig. 13) has a false labeling of about $20 \%$, where the mosaic could be reconstructed perfectly with the right ellipsoidal germs. The reason for this might be the low amount of germs and cells, and the corresponding edge effect, or that the ellipsoidal germs are reconstructed from the inertia matrices of each region independently from the surrounding packets. The surrounding germs influence highly the cell structure and need to be taken into account for a better reconstruction. One possibility might be to iteratively adapt the ellipsoidal germs according to the reconstructed cells. Such an iterative approach stays in the perspectives of this work.

\section{CONCLUSION}

This paper has proven the necessity and advantage of anisotropic tessellation models to describe complex polycrystalline microstructures. We have proposed several metrics to define anisotropic models that are generalization of the classical Laguerre and JohnsonMehl tessellations. Furthermore, we have shown how to achieve reconstructions of real microstructures as anisotropic tessellation models. This procedure has been validated on several 2D EBSD images on martensitic steel, where it has shown a reconstruction error of under $10 \%$ false labeled pixels. These results have shown that the model is well fitted for the reconstruction of bainitic or martensitic lath packets in steel.

For the reconstruction of a 3D image, the model yields less degrees of freedom and has therefore a higher level of abstraction. The 3D reconstruction of artificial tessellations shows that a more complex algorithm might be required to minimize the false labeling. This is also known for isotropic models: the simple barycenter and volume adaption is not sufficient for an accurate reconstruction.

\section{PERSPECTIVES}

Further studies might concern the fitting of the stochastic model to geometrical characteristics of real microstructures. It is desirable to be able to estimate distribution parameters from 2D EBSD images to create realistic 3D realisations of the anisotropic tessellation model. Those realisations and their representation as surfacic or volumetric meshes yield the possibility to virtually study the mechanical and thermic behaviour of the material.

\section{ACKNOWLEDGEMENTS}

We thank EDF for supporting this project and for providing the 3D EBSD image, and Anne-Françoise Gourgues for her professional help and explanations.

\section{REFERENCES}

Albert S, Matsui M, Watanabe T, Hongo H, Kubo K, Tabuchi M (2003). Variation in the type IV cracking behaviour of a high $\mathrm{Cr}$ steel weld with post weld heat treatment. Int J Pres Ves Pip 80:405-13.

Blaschke W (1921). Vorlesungen über Differentialgeometrie III. Berlin: Springer.

Descartes R (1644). Les principes de la philosophie. Amsterdam: Louis Elzevier.

Dirichlet GL (1850). Über die Reduktion der positiven quadratischen Formen mit drei unbestimmten ganzen Zahlen. J Reine Angew Math 40:209-27.

Jeulin D (2013). Random tessellations and Boolean random functions. In: Hendriks C, Borgefors G, Strand R, eds. Mathematical morphology and its applications to signal and image processing. Lect Notes Comput Sci 7883:2536.

Johnson WA, Mehl RF (1939). Reaction kinetics in processes of nucleation and growth. Trans AIME 135:416-58.

Lautensack C (2007). Random Laguerre tessellations. PhD Thesis, Universität Karlsruhe (TH).

McCartin BJ (2007). On concentration and inertia ellipsoids. Appl Math Sci 1:1-11.

Vivier F (2009). Fluage à $500 \mathrm{C}$ d'un joint soudé d'un acier 9Cr-1Mo modifié: évolution de la microstructure et comportement mécanique. PhD thesis, ParisTech MAT- Centre des matériaux PM Fourt.

Voronoi G (1908). Nouvelles applications des paramètres continus à la théorie des formes quadratiques. premier mémoire. Sur quelques propriétés des formes quadratiques positives parfaites. J Reine Angew Math 133:97-102.

\section{APPENDIX: PROOF OF THE EQUIVALENCE OF TESSELLATIONS}

We will show that tessellations with metrics $d_{e}, d_{1}, d_{2}$ are equivalent. We assume that $p \in \mathbb{R}^{3}$ lies on a boundary between the ellipsoidal germs $e_{1}$ and $e_{2}$ under the metric $d_{e}$. This implies that 


$$
\begin{aligned}
& d_{e}^{2}\left(p, e_{1}\right)=d_{e}^{2}\left(p, e_{2}\right) \\
\Rightarrow & d_{e}^{2}\left(p, e_{1}\right)-1=d_{e}^{2}\left(p, e_{2}\right)-1 \\
\Rightarrow & d_{1}^{2}\left(p, e_{1}\right)=d_{1}^{2}\left(p, e_{2}\right) \\
(7) \Rightarrow & d_{e}\left(p, e_{1}\right)=d_{e}\left(p, e_{2}\right) \\
\Rightarrow & d_{e}\left(p, e_{1}\right)-1=d_{e}\left(p, e_{2}\right)-1 \\
\Rightarrow & d_{2}\left(p, e_{1}\right)=d_{2}\left(p, e_{2}\right)
\end{aligned}
$$

And therefore $p$ also lies on a boundary between the germs $e_{1}$ and $e_{2}$ under the metrics $d_{1}$ and $d_{2}$. It means that the set of boundaries $B_{e}$ generated under metric $d_{e}$ is a subset of $B_{1}$ and $B_{2}$ (the set of boundaries under metric $d_{1}$ and $d_{2}$ ). Vice versa, we can show that $B_{1}, B_{2} \subset B_{e}$ and therefore $B_{e}=B_{1}=B_{2}$. 